This document is published in:

IEEE Transactions on Vehicular Technology 62 (2013), 8-Oct, pp. 3653 - 3662

DOI: 10.1109/TVT.2013.2260571

(C) 2013 IEEE. Personal use of this material is permitted. Permission from IEEE must be obtained for all other uses, in any current or future media, including reprinting/republishing this material for advertising or promotional purposes, creating new collective works, for resale or redistribution to servers or lists, or reuse of any copyrighted component of this work in other works. 


\title{
Iterative Joint Estimation Procedure for Channel and Frequency Offset in Multi-Antenna OFDM Systems With an Insufficient Cyclic Prefix
}

\author{
Carlos Prieto del Amo and M. Julia Fernández-Getino García, Member, IEEE
}

\begin{abstract}
This paper addresses a strategy to improve the joint channel and frequency offset (FO) estimation in multiantenna systems, widely known as multiple-input-multiple-output orthogonal frequency-division multiplexing (MIMO-OFDM), in the presence of intersymbol interference (ISI) and intercarrier interference (ICI) occasioned by an insufficient cyclic prefix (CP). The enhancement is attained by the use of an iterative joint estimation procedure (IJEP) that successively cancels the interferences located in the preamble of the OFDM frame, which is used for the joint estimation and initially contains the interferences due to a CP shorter than the channel length. The IJEP requires at certain steps a proper iterative interference cancellation algorithm, which makes use of an initial FO compensation and channel estimation obtained due to the use of a symmetric sequence in the preamble. After the iterative cancellation of interferences, the procedure performs an additional joint channel and FO estimation whose mean square error converges to the Cramér-Rao bound (CRB). Later on, this subsequent joint estimation permits the removal of the interferences in the data part of the frame, which are also due to an insufficient $\mathrm{CP}$, in the same iterative fashion but saving iterations compared with the use of other estimation strategies. The appraisal of the procedure has been performed by assessing the convergence of the simulated estimators to the CRB as a function of the number of iterations. Additionally, simulations for the evaluation of the bit error rate (BER) have been carried out to probe how the utilization of the proposed IJEP clearly improves the performance of the system. It is concluded that, with a reduced number of iterations in the preamble, the IJEP converges to the theoretical bounds, thus reducing the disturbances caused by a hard wireless channel or a deliberately insufficient CP. Therefore, this eases the interference cancellation in the data part, leading to an improvement in the BER that approximates to the ideal case of a sufficient $\mathrm{CP}$ and, consequently, an improvement in the computational cost of the whole procedure that has been analyzed.
\end{abstract}

Index Terms-Channel estimation, frequency offset (FO) estimation, insufficient cyclic prefix (CP), interference cancellation, multiple-input-multiple-output orthogonal frequencydivision multiplexing (MIMO-OFDM).

\section{INTRODUCTION}

$\mathbf{N}$ OWADAYS, communications are suffering an important change, considering the increasing global mobile penetration rate and the way the users access the resources offered

This work was supported in part by the Spanish na-tional projects GRE3N-SYST (TEC2011-29006-C03-03) and COMONSENS (CSD2008-00010).

The authors are with the Department of Signal Theory and Communications, Carlos III University of Madrid, 28911 Leganés, Spain (e-mail: cprieto@tsc. uc3m.es; mjulia@tsc.uc3m.es). by the service providers. Currently, the users are demanding an augment in the system bandwidth since the new applications and services require it this way. In addition, this has to be done independently of the user location and, consequently, the channel seen from the terminals. Clear examples are the dramatic increment in the usage of mobile terminals as if they were office, domestic, or vehicular users; the utilization of Digital Video Broadcasting services through handheld and vehicular terminals [1]; the access to in-demand technologies such as high-definition television [2], in both fixed and mobile devices [3]; and many other instances that we all are experiencing continuously.

These facts entail the development of modern techniques to enhance spectral efficiency and network performance to provide effective higher bandwidths. One of the ways to meet these constraints was, and currently still is, the inclusion of orthogonal frequency-division multiplexing (OFDM) in most of the communications standards [4], [5] due to its intrinsic features [6] and the maturity reached by this technology. However, the necessity of a continuous increase in the capacity of the communications systems led to the incorporation of several terminals between the transmitter and the receiver, which is widely known as multiple-input-multiple-output (MIMO) systems [7].

Commonly, the OFDM technology makes use of a guard period named cyclic prefix $(\mathrm{CP})$, whose length $L_{\mathrm{CP}}$ has to be longer than the channel length $L$ to ensure a proper system performance. This CP implies a decrease in the capacity of the system since it does not incorporate additional information. This is the reason why its suppression, which leads to an increase in capacity, is currently under thorough analysis as the work disclosed in this paper. On the other hand, the access to communications services independently of the user location drives to a length of the communication channel longer than the $\mathrm{CP}$ in some particular cases. These two situations, which are considered in the sequel as a scenario of an insufficient CP, give rise to similar impairments in the system.

What an insufficient CP implies is the appearance of intersymbol interference (ISI) and intercarrier interference (ICI) [8]-[13] that must be mitigated or canceled to achieve a proper system performance. This has been already dealt with in the literature, in both MIMO [9] and single-input single-output [10][13], where some iterative interference cancellation algorithms [10], [11] or filtering procedures [12], [13] are introduced. Nevertheless, these works usually consider ideal conditions for the estimation of parameters that ease the mitigation of the interferences in the data part. For instance, in [10] and [11], 
the channel estimator that is used for iterative cancellation of disturbances is attained for a case where the preamble always fulfills the $\mathrm{CP}$ condition, that is, $L_{\mathrm{CP}}$ longer than $L$. Consequently, the insufficient $\mathrm{CP}$ scenario is only deemed in the data signal, and the interference cancellation is performed uniquely in this part. The non-consideration of an insufficient $\mathrm{CP}$ in the preamble is usually tackled in the current standards [14], where up to one fourth of the frame length can be used as the CP. Despite this fact, either hard channel conditions or partial and total suppression of the CP to increase the capacity can lead to channel lengths surpassing the reserved $\mathrm{CP}$ and thus give rise to interferences in the preamble as well.

The appearance of ISI and ICI due to an insufficient CP in the preamble has been coped with in some works [15][17], but the method of handling this issue drives to nonoptimal estimators and does not converge to the Cramér-Rao bound (CRB) and, therefore, does not permit an interference cancellation procedure in the data part that is as effective as it could be, due to inaccurate channel estimations. In fact, in [15][17], a symmetric sequence (SS) in the time domain, which is an OFDM symbol with two equal halves, is employed in the preamble. This SS mostly absorbs ISI and ICI in the first half, which is discarded, enabling the estimation with only the second half. Although simple, this estimation strategy relatively works but ignores the information of the first half, which is the reason why its performance does not approach the CRB corresponding to the whole OFDM symbol, that is, bearing in mind both halves.

Additionally, the frequency offset (FO) impairment must be considered as part of the system since it is present in real scenarios, and thus, it has been dealt with in a previous reference analyzing the channel estimation where there is an insufficient CP in the preamble part [17].

In this paper, we introduce a novel iterative joint FO and channel estimation algorithm that uses a preamble affected by an insufficient $\mathrm{CP}$, whose main contributions are explained next.

First, our proposal addresses the joint estimation of both parameters, i.e., channel and FO, in the scenarios of concern. Most previous contributions only describe channel estimation and insufficient $\mathrm{CP}$, excluding the effect of FO, and cope with ideal conditions in the preamble symbols, that is, they do not analyze the effect of distortions within the preamble to perform proper joint estimations. In fact, only the previous work in [17] addressed the joint estimation.

Second, in this paper, the joint estimation is based on all the samples of the preamble ( $N$ samples), despite the fact that the first half ( $N / 2$ samples) conveys ISI-ICI. Previous works [15]-[17] only rely on the second half of the preamble $(N / 2$ samples) to perform the estimation. Our work yields a quasioptimal channel and FO estimation that approaches to the CRB of the whole symbol, unlike previous proposals [15]-[17]. The algorithm is an iterative joint estimation procedure (IJEP) that successively cancels the interferences located in the preamble part of the OFDM frame. We propose an algorithm of six steps that first, in steps 1 and 2, attains an initial FO compensation and channel estimation obtained due to the utilization of an SS as preamble. This initial estimation permits the development of an iterative interference cancellation algorithm (step 4) in the part of the preamble, including most of the distortion, which later on makes possible the attainment of a subsequent joint estimation whose mean square error (MSE) converges to the CRB.

Third, after the joint estimation of both parameters based on the preamble, those estimations are used in the data symbols behind the preamble. Since the estimates converge to the bounds, the iterative interference cancellation in the data part of the frame, which also has an insufficient $\mathrm{CP}$, is performed more efficiently since, as we provide a more accurate estimation of both parameters, the number of iterations needed to mitigate the interferences in the data part is less than if other estimation strategies were employed. The assessment of the system by simulations shows an appreciable improvement in the bit error rate (BER) of the whole system. Our proposal clearly outperforms existing works [15]-[17] while converging to the ideal case of a sufficient CP, which is denoted by SCP in the sequel.

Finally, a detailed analysis of the computational cost of the procedure disclosed in Section $\mathrm{V}$ probes the cost saving in number of required cycles of a digital signal processor (DSP) and, therefore, the extension of battery duration and reduction in latency time.

The remainder of this paper is organized as follows. Section II presents the signal model and the joint maximumlikelihood (ML) estimator. Next, Section III introduces the proposed iterative estimation procedure IJEP. The theoretical bounds to assess the simulations are defined by CRBs specified in Section IV. In Section V, an analysis of the computational cost is included, whereas the simulation results are provided in Section VI. Finally, in Section VII, some conclusions are drawn.

Notation: Uppercase (lowercase) boldface letters express matrices (vectors), $(\cdot)^{H}$ represents Hermitian operation and $(\cdot)^{T}$ the transpose, $|\cdot|$ means absolute value, $\mathbf{0}_{M \times N}$ is an $M \times N$ null matrix, $\mathbf{I}_{N}$ is an identity matrix of size $N, \operatorname{diag}\{\mathbf{x}\}$ is a diagonal matrix with $\mathbf{x}$ as the elements of its main diagonal and zero elsewhere, $\langle\cdot\rangle_{N}$ stands for the modulo- $N$ operation, and $\operatorname{Re}(\cdot)$ and $\operatorname{Im}(\cdot)$ account for the real and imaginary parts, respectively. We will refer to $\mathbf{x}$ as time-domain vectors and to $\tilde{\mathbf{x}}$ as frequency-domain vectors.

\section{SignAl MODEL}

Here, a typical MIMO-OFDM system will be considered with $N_{t}$ and $N_{r}$ transmit and receive antennas, respectively. The signal model in this multi-antenna scenario is constructed by utilizing the time-domain symbols, which are obtained from the frequency-domain symbols using an $N$-length inverse discrete Fourier transform (IDFT) operation, being $N$ the number of subcarriers. The insufficient $\mathrm{CP}$ condition addressed in this paper introduces two additional terms in the general expression of the received signal. Consequently, once the $\mathrm{CP}$ has been removed, the received signal at the $j$ th antenna $j=1,2, \ldots N_{r}$ at the $\ell$ th OFDM symbol time can be formulated as [17]

$$
\tilde{\mathbf{r}}_{j}(\ell)=\mathbf{C}_{o}(\varepsilon)\left[\sum_{i=1}^{N_{t}} \mathbf{F}_{N} \mathbf{H}_{j i} \mathbf{F}_{N}^{H} \tilde{\mathbf{x}}_{i}(\ell)\right.
$$




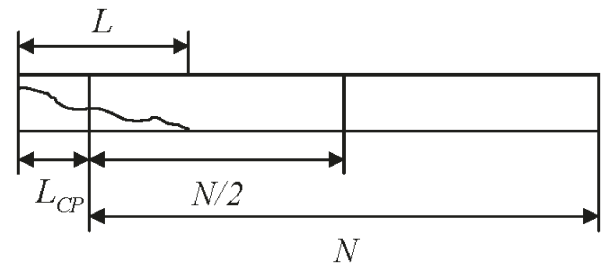

Fig. 1. Channel spreading over an SS in the time domain.

$$
\begin{aligned}
& +\sum_{i=1}^{N_{t}} \mathbf{F}_{N} \mathbf{H}_{j i}^{\mathrm{ISI}} \mathbf{F}_{N}^{H} \tilde{\mathbf{x}}_{i}(\ell-1) \\
& \left.-\sum_{i=1}^{N_{t}} \mathbf{F}_{N} \mathbf{H}_{j i}^{\mathrm{ICI}} \mathbf{F}_{N}^{H} \tilde{\mathbf{x}}_{i}(\ell)\right]+\tilde{\mathbf{w}}_{j}(\ell)
\end{aligned}
$$

where $\tilde{\mathbf{r}}_{j}(\ell)$ is an $N \times 1$ vector with the frequency-domain received signal; $\mathbf{F}_{N}$ is the discrete Fourier transform matrix of size $N \times N ; \mathbf{C}_{o}(\varepsilon)=\operatorname{diag}\left\{e^{(0, \iota 2 \pi \varepsilon, \ldots, \iota 2 \pi \varepsilon(N-1))}\right\}$ encompasses the FO, being $\varepsilon$ the FO normalized by the subcarrier spacing $\Delta f$ and $\iota=\sqrt{-1} ; \mathbf{H}_{j i}$, with $i=1,2, \ldots, N_{t}$ and $j=1,2, \ldots, N_{r}$, is an $N \times N$ circulant matrix, which consists of the channel impulse responses (CIRs) $\mathbf{h}_{j i}$ of size $L \times 1$ between the $i$ th transmit and the $j$ th receive antennas, where $L$ denotes the channel length, and each element $(s, t)$ of the matrix $\left[\mathbf{H}_{j i}\right]_{s, t}$ is given by $\mathbf{h}_{j i,\langle s-t\rangle_{N}}$, with $0 \leq s \leq N-1$ and $0 \leq t \leq N-1 ; \tilde{\mathbf{x}}_{i}(\ell)$ represents an $N \times 1$ vector with the transmitted signal from the $i$ th antenna; the ISI and ICI disturbances occasioned by the previous and current symbols $\tilde{\mathbf{x}}_{i}(\ell-1)$ and $\tilde{\mathbf{x}}_{i}(\ell)$ are given by $\mathbf{H}_{j i}^{\mathrm{ISI}}$ and $\mathbf{H}_{j i}^{\mathrm{ICI}}$, respectively; finally, $\tilde{\mathbf{w}}_{j}(\ell)$ accounts for the $N \times 1$ Gaussian noise component with zero mean and $\sigma_{n}^{2}$ variance, at the $j$ th receive antenna at the $\ell$ th symbol time. The desired signal is denoted by the first term between brackets in (1), whereas the second and third terms describe the ISI and ICI components, respectively.

\section{A. Symmetric Sequences (SS)}

We will consider that the preamble, which consists of a complete OFDM symbol, is constructed with an SS in the time domain, as some of the current standards and proposals do [18]. The SS is obtained with the transmission of the pilot sequence on the even subcarriers, whereas zeros are used on the odd subcarriers, driving to a symbol with two identical halves in the time domain. The way to exploit this $N / 2$ periodicity of an SS to reduce the effects of the insufficient $\mathrm{CP}$ consists in discarding at reception the first half of the SS, which is affected by ISI and ICI, and using only the second part for an initial channel and FO estimation (assuming $L<N / 2$ ). Fig. 1 shows the actual effect of the interferences within the preamble built with an SS.

The SS can convey optimal values as those proposed in [16] or known pseudonoise (PN) sequences in the frequency domain, named PNSS in [17]. The aim is to maintain the orthogonality between the multiple antennas. With PNSS, the multiple antennas employ totally different PN sequences with the advantage that PNSS outperforms other SS previously proposed in terms of the FO acquisition range, as shown in [17].

The expression of the PNSS for the $i$ th transmit antenna is given by

$$
\tilde{\mathbf{x}}_{i, k}= \begin{cases}\alpha e^{\iota \mathbf{c}_{i, k}}, & k \in \text { even } \\ 0, & k \in \text { odd }\end{cases}
$$

where $\tilde{\mathbf{x}}_{i, k}$ is the $k$ th element of the transmitted preamble, where $k=0, \ldots, N-1$ is the subcarrier index, $\alpha$ is a constant amplitude, and $\mathbf{c}_{i, k}$ is the $k$ th element of the $i$ th PN sequence $\mathbf{c}_{i}$ of length $N$, which randomly modulates the phase of the signal for the even subcarriers.

At the receiver side, the ISI and ICI contributions are mostly absorbed by the first half of the SS; hence, the corresponding second and third terms in (1) can be considered null if the first half is discarded. Consequently, the expression (1) corresponding to the preamble can be converted to the time domain as

$$
\mathbf{r}_{j}(\ell)=\mathbf{C}_{o}(\varepsilon)\left[\sum_{i=1}^{N_{t}} \mathbf{H}_{j i} \mathbf{x}_{i}(\ell)\right]+\mathbf{w}_{j}(\ell)
$$

where $\mathbf{r}_{j}(\ell)=\mathbf{F}_{N}^{H} \tilde{\mathbf{r}}_{j}(\ell)$ and $\mathbf{x}_{i}(\ell)=\mathbf{F}_{N}^{H} \tilde{\mathbf{x}}_{i}(\ell)$ are two $N \times 1$ vectors with the time-domain received and transmitted signals after the IDFT operation, respectively. Hereafter, the symbol time index $\ell$ will be omitted in the sequel for the sake of clarity.

The operation of discarding the first half implies considering only the second $N / 2$ samples of (3) so that the expression of the second half of the received preamble can be expressed in matrix form as

$$
\mathbf{r}_{N / 2}=\mathbf{C}_{N / 2}(\varepsilon) \mathbf{X}_{N / 2} \mathbf{h}+\mathbf{w}_{N / 2}
$$

where now $\mathbf{r}_{N / 2}$ is an $\left(\frac{N}{2} N_{r}\right) \times 1$ vector whose $j$ th element $\mathbf{r}_{N / 2, j}$, with $j=1,2, \ldots, N_{r}$, is the received signal at the $j$ th receive antenna given by a column $N / 2$-vector; $\mathbf{C}_{N / 2}(\varepsilon)$ is an $\left(\frac{N}{2} N_{r}\right) \times\left(\frac{N}{2} N_{r}\right)$ diagonal matrix with the submatrix $\mathbf{C}_{o, N / 2}(\varepsilon)$ in its main diagonal defined as

$$
\mathbf{C}_{N / 2}(\varepsilon)=\left[\begin{array}{cccc}
\mathbf{C}_{O, N / 2}(\varepsilon) & \mathbf{0}_{\frac{N}{2} \times \frac{N}{2}} & \cdots & \mathbf{0}_{\frac{N}{2} \times \frac{N}{2}} \\
\mathbf{0}_{\frac{N}{2} \times \frac{N}{2}} & \mathbf{C}_{o, N / 2}(\varepsilon) & \ddots & \mathbf{0}_{\frac{N}{2} \times \frac{N}{2}} \\
\vdots & \vdots & \ddots & \vdots \\
\mathbf{0}_{\frac{N}{2} \times \frac{N}{2}} & \mathbf{0}_{\frac{N}{2} \times \frac{N}{2}} & \cdots & \mathbf{C}_{o, N / 2}(\varepsilon)
\end{array}\right]
$$

and $\mathbf{C}_{o, N / 2}(\varepsilon)=\operatorname{diag}\left\{e^{(\iota 2 \pi \varepsilon N / 2, \iota 2 \pi \varepsilon(N / 2+1), \ldots, \iota 2 \pi \varepsilon(N-1))}\right\}$; the column vector $\mathbf{h}$ of length $\left(N_{t} N_{r} L\right)$ collects all the CIRs $\mathbf{h}_{j i}$; and the matrix $\mathbf{X}_{N / 2}$ is built as indicated in (6), shown at the bottom of the next page, where $\mathbf{X}_{N / 2, i}$ is an $\frac{N}{2} \times L$ circulant matrix for the $i$ th transmitter, where the entry $(s, t)$ is given by $\mathbf{x}_{N / 2, i,\langle s-t\rangle_{N / 2}}$, with $0 \leq s \leq N / 2-1$, $0 \leq t \leq L-1$, and $\mathbf{x}_{N / 2, i}$ being the last $N / 2$ samples of $\mathbf{x}_{i}$. The utilization of $\mathbf{0}_{\frac{N}{2} \times L}$ in (6) is due to the consideration of a multiple-antenna scenario.

Similarly, if initially there were no interferences in the received signal corresponding to the preamble because the $\mathrm{CP}$ is longer than the channel length or, as in our case, the interferences have been mostly canceled, the matrix form for the received whole preamble is given by

$$
\mathbf{r}=\mathbf{C}(\varepsilon) \mathbf{X h}+\mathbf{w}
$$

where it is built in the same fashion as (4) but considering in this occasion $N$ samples for all its components. This last expression will be used in the derivations of the ML estimation in Section II-B and the CRB in Section IV. 


\section{B. ML Estimation}

For the ML estimator, the resultant likelihood function

$$
\begin{aligned}
\mathbf{\Lambda}(\mathbf{r} \mid \mathbf{h})=\frac{1}{\left(\pi \sigma_{n}^{2}\right)^{N}} & \exp \left\{-\frac{1}{\sigma_{n}^{2}}\right. \\
& \left.\times\left(|\mathbf{r}-\mathbf{C}(\varepsilon) \mathbf{X h}|^{H}|\mathbf{r}-\mathbf{C}(\varepsilon) \mathbf{X h}|\right)\right\}
\end{aligned}
$$

corresponding to the received signal of either the whole preamble (7) or the second half (4) must be solved to attain the ML estimation of the channel $\mathbf{h}$ and the $\mathrm{FO} \varepsilon$ given, respectively, by

$$
\begin{aligned}
\hat{\mathbf{h}} & =\left(\mathbf{X}^{H} \mathbf{X}\right)^{-1} \mathbf{X}^{H} \mathbf{C}^{H}(\varepsilon) \mathbf{r}=\mathbf{A} \mathbf{X}^{H} \mathbf{C}^{H}(\varepsilon) \mathbf{r} \\
J(\varepsilon) & =\mathbf{r}^{H} \mathbf{C}(\varepsilon) \mathbf{X}\left(\mathbf{X}^{H} \mathbf{X}\right)^{-1} \mathbf{X}^{H} \mathbf{C}^{H}(\varepsilon) \mathbf{r} \\
& =\mathbf{r}^{H} \mathbf{C}(\varepsilon) \mathbf{P} \mathbf{C}^{H}(\varepsilon) \mathbf{r}
\end{aligned}
$$

with $\mathbf{A}=\left(\mathbf{X}^{H} \mathbf{X}\right)^{-1}$ and $\mathbf{P}=\mathbf{X}\left(\mathbf{X}^{H} \mathbf{X}\right)^{-1} \mathbf{X}^{H}$. The estimation of $\varepsilon$ using the cost function $J(\varepsilon)$ can be solved by Newton's method, as indicated in [17]. Once the FO has been estimated, the solution is compensated in (9) to obtain the channel estimation.

It is worth noting that (9) and (10) must be properly modified if the estimators stem from (4), with regard to only the second half of the preamble.

\section{Iterative Joint Estimation Procedure}

The joint ML channel and FO estimation has been formerly performed in almost ideal conditions when a significant interference in the part of the preamble used for the estimation does not exist [15]-[17], that is, the first half of the SS is discarded, and the second half is used for the estimation. In fact, [17] showed that using the whole SS (both halves) yields an irreducible error floor. Therefore, we propose the implementation of the IJEP in the case of an insufficient CP scenario, using the $N$-length received signal (both halves), issue not tackled up to now. The complete procedure allows obtaining the two estimators jointly due to the interference cancellation performed in the preamble. The procedure makes use of (9) and (10) introduced in the previous section to get the estimations and one of the interference cancellation algorithms referenced in the literature [16] to remove the interferences due to a channel length longer than the $\mathrm{CP}$.

The benefits of this novel iterative procedure are twofold. First, it provides a quasi-optimal channel and FO estimation. Second, this improved estimation of the parameters leads to a better performance in the data part. Since the data part also requires the execution of an iterative interference cancellation algorithm due to an insufficient $\mathrm{CP}$, which is also shorter than the channel length $L$, we can save iterations by the utilization of more accurately estimated system parameters.

The proposed IJEP is summarized in the following list, and the corresponding steps are explained next.

1) computation of $\hat{\varepsilon}_{1 / 2}$;

2) computation of $\hat{\mathbf{h}}_{1 / 2}$;

3) compensation of $\hat{\varepsilon}_{1 / 2}$ in the preamble;

4) interference cancellation in the preamble;

5) reinsertion of $\hat{\varepsilon}_{1 / 2}$ in the preamble;

6) computation of $\hat{\varepsilon}$ and $\hat{\mathbf{h}}$.

For the application of this iterative strategy, it is necessary to employ the particular structure of an SS in the preamble, as presented in Section II-A, since it enables proper mitigation of the ISI and ICI in the first four steps of the strategy by discarding the first half of the preamble.

1) Computation of $\hat{\varepsilon}_{1 / 2}$ : The initial step of the procedure is to calculate a prime $\mathrm{FO}$ estimation using the ML estimator

$$
\hat{\varepsilon}_{1 / 2}=\max _{\varepsilon} J(\varepsilon)
$$

where

$$
\begin{aligned}
J(\varepsilon)=\mathbf{r}_{N / 2}^{H} \mathbf{C}_{N / 2}(\varepsilon) \mathbf{X}_{N / 2} \\
\quad \times\left(\mathbf{X}_{N / 2}^{H} \mathbf{X}_{N / 2}\right)^{-1} \mathbf{X}_{N / 2}^{H} \mathbf{C}_{N / 2}^{H}(\varepsilon) \mathbf{r}_{N / 2} .
\end{aligned}
$$

As explained above, to overcome the effect of the interferences, the first half of the preamble is discarded at reception, which is denoted in (12) by $\mathbf{r}_{N / 2}$, that accounts for the last $N / 2$ samples of $\mathbf{r}$. Iteratively solving the cost function (12), we obtain the initial estimation $\hat{\varepsilon}_{1 / 2}$.

2) Computation of $\hat{\mathbf{h}}_{1 / 2}$ : After the initial FO estimation, the subsequent channel estimation is attained by

$$
\hat{\mathbf{h}}_{1 / 2}=\left(\mathbf{X}_{N / 2}^{H} \mathbf{X}_{N / 2}\right)^{-1} \mathbf{X}_{N / 2}^{H} \mathbf{C}_{N / 2}^{H}\left(\hat{\varepsilon}_{1 / 2}\right) \mathbf{r}_{N / 2}
$$

which makes use of the elements defined in step 1 and introduces the value of the estimated $\hat{\varepsilon}_{1 / 2}$ in submatrix $\mathbf{C}_{o, N / 2}\left(\hat{\varepsilon}_{1 / 2}\right)$ [see (5)].

3) Compensation of $\hat{\varepsilon}_{1 / 2}$ in the Preamble: Before canceling the interferences, it is necessary to compensate $\hat{\varepsilon}_{1 / 2}$ in the whole preamble, which was previously obtained in step 1 . This is performed through the expression

$$
\mathbf{r}_{j}^{\prime}=\mathbf{r}_{j} \exp \left\{\left(0, \iota 2 \pi \hat{\varepsilon}_{1 / 2}, \ldots, \iota 2 \pi \hat{\varepsilon}_{1 / 2}(N-1)\right)^{T}\right\} .
$$

$$
\mathbf{X}_{N / 2}=\left[\begin{array}{ccccccccccccc}
\mathbf{X}_{N / 2,1} & \mathbf{X}_{N / 2,2} & \cdots & \mathbf{X}_{N / 2, N_{t}} & \mathbf{0}_{\frac{N}{2} \times L} & \mathbf{0}_{\frac{N}{2} \times L} & \cdots & \mathbf{0}_{\frac{N}{2} \times L} & \cdots & \mathbf{0}_{\frac{N}{2} \times L} & \mathbf{0}_{\frac{N}{2} \times L} & \cdots & \mathbf{0}_{\frac{N}{2} \times L} \\
\mathbf{0}_{\frac{N}{2} \times L} & \mathbf{0}_{\frac{N}{2} \times L} & \cdots & \mathbf{0}_{\frac{N}{2} \times L} & \mathbf{X}_{N / 2,1} & \mathbf{X}_{N / 2,2} & \cdots & \mathbf{X}_{N / 2, N_{t}} & \cdots & \mathbf{0}_{\frac{N}{2} \times L} & \mathbf{0}_{\frac{N}{2} \times L} & \cdots & \mathbf{0}_{\frac{N}{2} \times L} \\
\vdots & \vdots & \ddots & \vdots & \vdots & \vdots & \ddots & \vdots & \ddots & \vdots & \vdots & \ddots & \vdots \\
\mathbf{0}_{\frac{N}{2} \times L} & \mathbf{0}_{\frac{N}{2} \times L} & \cdots & \mathbf{0}_{\frac{N}{2} \times L} & \mathbf{0}_{\frac{N}{2} \times L} & \mathbf{0}_{\frac{N}{2} \times L} & \cdots & \mathbf{0}_{\frac{N}{2} \times L} & \cdots & \mathbf{X}_{N / 2,1} & \mathbf{X}_{N / 2,2} & \cdots & \mathbf{X}_{N / 2, N_{t}}
\end{array}\right]
$$


In this way, the distortion introduced in the system by the FO is removed. Before the next step, the received signal needs to be converted to the frequency domain, i.e., $\tilde{\mathbf{r}}_{j}=\mathbf{F}_{N} \mathbf{r}_{j}^{\prime}$.

4) Interference Cancellation in the Preamble: In this step, an algorithm for interference cancellation is utilized to mitigate the distortion introduced due to an insufficient CP [16]. The process consists in subtracting and compensating certain components corresponding to the ISI and ICI. Considering (1), this received signal in the frequency domain at the $j$ th antenna can be written in reduced form by

$$
\tilde{\mathbf{r}}_{j}=\sum_{i=1}^{N_{t}} \tilde{\mathbf{r}}_{j, i}^{U}+\sum_{i=1}^{N_{t}} \tilde{\mathbf{r}}_{j, i}^{\mathrm{ISI}}-\sum_{i=1}^{N_{t}} \tilde{\mathbf{r}}_{j, i}^{\mathrm{ICI}}+\tilde{\mathbf{w}}_{j}
$$

where $\tilde{\mathbf{r}}_{j, i}^{U}$ is the useful term, and $\tilde{\mathbf{r}}_{j, i}^{\mathrm{ISI}}$ and $\tilde{\mathbf{r}}_{j, i}^{\mathrm{ICI}}$ are the ISI and ICI components, respectively. The process of cancellation is iteratively carried out and mainly consists of a cancellation of the ISI, labeled $C_{\mathrm{ISI}}$, by

$$
\tilde{\mathbf{r}}_{j}^{C_{\mathrm{ISI}}}=\tilde{\mathbf{r}}_{j}-\sum_{i=1}^{N_{t}} \tilde{\mathbf{r}}_{j, i}^{\mathrm{ISI}}
$$

and a subsequent compensation of the ICI, labeled $C_{\mathrm{ICI}}$, by

$$
\tilde{\mathbf{r}}_{j}^{C_{\mathrm{ICI}}}=\tilde{\mathbf{r}}_{j}^{C_{\mathrm{ISI}}}+\sum_{i=1}^{N_{t}} \mathbf{C}_{j, i}
$$

through the use of the ICI compensation term $\mathbf{C}_{j, i}$ that satisfies the following relation:

$$
\mathbf{C}_{j, i}+\tilde{\mathbf{r}}_{j, i}^{U}-\tilde{\mathbf{r}}_{j, i}^{\mathrm{ICI}}=\tilde{\mathbf{x}}_{i} \mathbf{F}_{N} \mathbf{H}_{j i} .
$$

This equality represents the general condition for ICI compensation so that it is possible to recover the transmitted signal simply if $\tilde{\mathbf{x}}_{i}$ is multiplied by the frequency-domain channel matrix, provided that the ICI compensation term is added to the received signal. ${ }^{1}$ Consequently, after having obtained $\tilde{\mathbf{r}}_{j}^{C_{\mathrm{ICI}}}$ and having properly estimated the channel matrix $\mathbf{H}_{j i}$, it is possible to attain the transmitted symbol $\tilde{\mathbf{x}}_{i}$.

5) Reinsertion of $\hat{\varepsilon}_{1 / 2}$ in the Preamble: Once the interferences have been canceled in the preamble, we can estimate again the FO and the channel, but it is necessary this step that consists in the reinsertion of the previously estimated FO, i.e., $\hat{\varepsilon}_{1 / 2}$, in the preamble. This is performed similarly as in step 3 using

$$
\mathbf{r}_{j}^{\prime C_{\mathrm{ICI}}}=\mathbf{r}_{j}^{C_{\mathrm{ICI}}} \exp \left\{-\left(0, \iota 2 \pi \hat{\varepsilon}_{1 / 2}, \ldots, \iota 2 \pi \hat{\varepsilon}_{1 / 2}(N-1)\right)^{T}\right\}
$$

and considering $\mathbf{r}_{j}^{C_{\mathrm{ICI}}}=\mathbf{F}_{N}^{H} \tilde{\mathbf{r}}_{j}^{C_{\mathrm{ICI}}}$.

6) Computation of $\hat{\varepsilon}$ and $\hat{\mathbf{h}}$ : This step encompasses the usual joint FO and channel estimation described in (9) and (10) but using $\mathbf{r}_{j}^{\prime C_{\mathrm{ICI}}}$.

The whole procedure of the iterative joint estimation is shown in Fig. 2 in the lower branch of the block diagram. This

\footnotetext{
${ }^{1}$ This expression considers that the ISI term has been already subtracted and that there is no noise in the system.
}

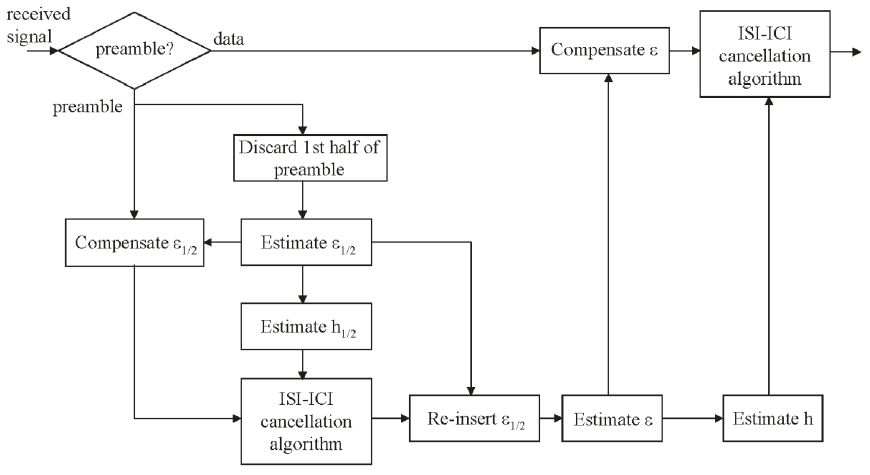

Fig. 2. Block diagram of the proposed IJEP.

branch is aimed at performing the estimation and cancellation of interferences in the preamble. Once the adequate estimations have been obtained, the data symbols suffering the effects of an insufficient $\mathrm{CP}$ are led through the upper branch to compensate the FO and mitigate the interferences with the same algorithm as that utilized in step 4 of the proposed procedure.

\section{Performance Analysis}

The performance analysis in the simulations will be assessed by the CRB, both for FO and channel estimations. The computation of the CRB is derived from the Fisher information matrix (FIM), obtaining the derivatives of the log-likelihood function (8) of the received data with respect to the vector $\boldsymbol{\theta}=$ $\left[\operatorname{Re}(\mathbf{h})^{T} \operatorname{Im}(\mathbf{h})^{T} \varepsilon\right]$, whose dimensions are $\left(2 N_{t} N_{r} L+1\right) \times 1$. The $u$ th component of the CRB vector is found as the $[u, u]$ element of the inverse of the FIM or

$$
\operatorname{var}\left\{\boldsymbol{\theta}_{u}\right\} \geq\left[\mathbf{F I M}^{-1}\{\boldsymbol{\theta}\}\right]_{u u}=\mathbf{C R B}\left\{\boldsymbol{\theta}_{u}\right\}
$$

where $u$ is within the interval $[1,2,3]$ in this case. After some manipulations included in [17], the resulting form for the FIM matrix, with dimensions $\left(2 N_{t} N_{r} L+1\right) \times\left(2 N_{t} N_{r} L+1\right)$, yields (21), shown at the bottom of the next page, where $\mathbf{B}=\operatorname{diag}\{0,1, \ldots, N-1, \ldots, 0,1, \ldots, N-1\}$ is a diagonal matrix of size $\left(N_{r} N\right) \times\left(N_{r} N\right)$.

Using (20), we can obtain the CRB of an independent estimated parameter included in the parameter vector $\boldsymbol{\theta}$. Consequently, extracting the diagonal of the inverted FIM matrix, i.e., FIM $^{-1}(\boldsymbol{\theta})$, we get the CRB vector with the bounds for the $2 N_{t} N_{r} L+1$ parameters. The last element of the vector $\operatorname{CRB}(\theta)$ is the bound for the FO, whereas the rest of the elements correspond to the real and imaginary parts of the channel taps.

Due to the high number of independent channel parameters, their evaluation should be done individually, i.e., in the case of two transmit and receive antennas $\left(N_{t}=2\right.$ and $\left.N_{r}=2\right)$, we would need $8 L$ individual assessments. Nevertheless, we will perform the evaluation using the average given by

$$
\mathbf{C R B}\{\mathbf{h}\}=E\left\{\sum_{i=1}^{4 L} \operatorname{CRB}\left\{\boldsymbol{\theta}_{i}\right\}+\sum_{i=4 L+1}^{8 L} \operatorname{CRB}\left\{\boldsymbol{\theta}_{i}\right\}\right\}
$$


where $E\{\cdot\}$ denotes the expected value. As stated above, the $\mathrm{CRB}$ of the FO will be calculated as

$$
\operatorname{CRB}\{\varepsilon\}=\operatorname{CRB}\{8 L+1\} .
$$

It must be noted that (22) and (23) are the CRBs when it is considered the whole preamble ( $N$ samples), in lieu of only using its second half, which can be straightforwardly derived.

See [17] for more details on the derivation of the CRB expressions.

\section{Computational Cost Saving}

A computational cost saving of one iteration for each OFDM data symbol, as it will be shown with the simulation results, involves less power consumption, let alone the reduction in the latency. This may be of high interest in the case of a practical implementation, as it is envisaged in a future step of this research. Next, we will analyze the impact of saving one iteration considering a fixed-point DSP, whose parameters are summarized in Table I [21]. Certainly, the process of executing the IJEP in the preamble also implies some computational cost not required if other previously proposed estimators are performed. Albeit, in OFDM frames, one preamble is usually followed by numerous data symbols; therefore, the additional computational cost induced in the preamble is negligible in the overall computational cost. We have to consider that the most consuming step in the IJEP corresponds to the cancellation procedure, which is indicated in step 4 . Thus, as this step has to be carried out in the data part in any case, with either other previously proposed estimators or the IJEP, we will obtain a considerable computational cost saving if we avoid one iteration per OFDM symbol, provided that the number of symbols in the data part is much higher than the number of iterations performed in the preamble. It is worth noting that this is the usual case since an OFDM frame will consist of more than five data symbols, which is the selected number of iterations executed in the preamble to reach the CRB.

The assessment of the computational cost takes into account (16) and (17), where the ISI cancellation and ICI compensation terms were presented. The detailed expressions of the interference cancellation are omitted here for the sake of brevity. With them, we have carried out a thorough analysis to find out the number of operations per task. The results are summarized in Table II.

- Task 1: It performs the remodulation of the OFDM symbol, which is 16-quadrature-amplitude modulation (QAM) or 4-QAM, depending on the simulated scenario. This does not require a noticeable number of operations since the modulated data can be directly obtained from a lookup table or by direct digital synthesis techniques.
TABLE I

MAIN PARAMETERS FOR A FIXED-POINT DSP

\begin{tabular}{cc}
\hline Parameter & Value \\
\hline Computer/Processor cycle/Second & $0.33 \mathrm{~mA} / \mathrm{MHz}$ \\
Supply Voltage & $1.26 \mathrm{~V}$ \\
Energy/cycle & $415.8 \mathrm{pW} \mathrm{sec/cycle}$ \\
Cycles/N-point FFT & $306+5 \frac{N}{2} \log _{2}\left(\frac{N}{2}\right)$ \\
Cycles/Multiplication & $1 / 2$ \\
Cycles/Addition & $1 / 4$ \\
Cycles/Complex Multiplication & 3 \\
Cycles/Complex Addition & $1 / 2$ \\
\hline$\left.{ }^{*}\right)$ Cycles/ $X$-length min-index search & $17+X / 2$ \\
\hline$\left.{ }^{*}\right)$ Cycles/ $Y$-length max-value sear & $Y / 2+6$ \\
\hline
\end{tabular}

(*) $X$ and $Y$ can take any value.

- Tasks 2 and 3: These two additional tasks are in charge of trunking the time-domain channels between the transmit and receive antennas and their conversion to the frequency domain. The former simply consists of the extraction of the samples of the channel taps that give rise to the interferences; hence, no appreciable time consumption has been considered. The latter implies the execution of $\left(N_{t} N_{r}\right)$ fast Fourier transform operations, which yields

$$
N_{t} N_{r}\left(306+5 / 2 N \log _{2}(N / 2)\right) \quad \text { cycles. }
$$

- Tasks 4 and 5: Subsequently, two more tasks are needed concerning the calculation of the ISI component and ICI compensation terms. After the evaluation of the expressions, it has been found that the total number of cycles for these two tasks, as disclosed in Table II, are

$$
\begin{aligned}
\sum_{i=1}^{N_{t}} \tilde{\mathbf{r}}_{j, i}^{\mathrm{ISI}}(\ell) \Rightarrow N_{t} N_{r} N( & +\frac{1}{2}\left(L-L_{\mathrm{CP}}\right)+ \\
& +\frac{7}{2}\left(L-L_{\mathrm{CP}}\right) N \\
& \left.+\frac{47}{4}\left(L-L_{\mathrm{CP}}\right)^{2} N\right) \quad \text { cycles } \\
\sum_{i=1}^{N_{t}} \mathbf{C}_{j, i}(\ell) \Rightarrow N_{t} N_{r} N( & +\frac{1}{2}\left(L-L_{\mathrm{CP}}\right)+ \\
& +\frac{7}{2}\left(L-L_{\mathrm{CP}}\right) N \\
& \left.+\frac{43}{4}\left(L-L_{\mathrm{CP}}\right)^{2} N\right) \quad \text { cycles }
\end{aligned}
$$

respectively. As it was supposed, the most time-consuming tasks of the whole procedure correspond to these two tasks.

- Task 6: It is necessary to calculate the interference canceled signal $\tilde{\mathbf{r}}^{\mathrm{ICI}}(\ell)$ by $N_{r} N$ cycles. The demodulated

$$
\mathbf{F I M}=\frac{2}{\sigma_{n}^{2}}\left[\begin{array}{ccc}
\operatorname{Re}\left(\mathbf{X}^{H} \mathbf{X}\right) & -\operatorname{Im}\left(\mathbf{X}^{H} \mathbf{X}\right) & -2 \pi \operatorname{Im}\left(\mathbf{X}^{H} \mathbf{B X h}\right) \\
\operatorname{Im}\left(\mathbf{X}^{H} \mathbf{X}\right) & \operatorname{Re}\left(\mathbf{X}^{H} \mathbf{X}\right) & 2 \pi \operatorname{Re}\left(\mathbf{X}^{H} \mathbf{B X h}\right) \\
2 \pi \operatorname{Im}\left(\mathbf{h}^{H} \mathbf{X}^{H} \mathbf{B X}\right) & 2 \pi \operatorname{Re}\left(\mathbf{h}^{H} \mathbf{X}^{H} \mathbf{B X}\right) & 4 \pi^{2} \operatorname{Re}\left(\mathbf{h}^{H} \mathbf{X}^{H} \mathbf{B}^{2} \mathbf{X} \mathbf{h}\right)
\end{array}\right]
$$


TABLE II

Number of Operations PER TASK of THE INTERFERENCE CANCELlation Algorithm

\begin{tabular}{|c|c|c|c|c|c|c|c|c|}
\hline \multicolumn{2}{|r|}{ Task } & \multicolumn{7}{|c|}{ \# Cycles } \\
\hline 1) & Remodulation & \multicolumn{7}{|c|}{0} \\
\hline 2) & Trunk Channels & \multicolumn{7}{|c|}{0} \\
\hline 3) & Convert Trunked Channels to Frequency Domain & \multicolumn{7}{|c|}{$N_{t} N_{r}\left(306+5 / 2 N \log _{2}(N / 2)\right)$} \\
\hline & & $N_{t} N_{r} N\left(L-L_{C P}\right)$ & $x$ & $\sum$ & 1 & complex add. & $=$ & $1 / 2)$ \\
\hline & & $N_{t} N_{r} N$ & $\times$ & $\sum$ & 2 & complex mult. & $=$ & $6)$ \\
\hline 5) & Compute $\sum_{i=1}^{N_{t}} \mathbf{C}_{j, i}(\ell)$ & $N_{t} N_{r} N\left(L-L_{C P}\right) N\left(L-L_{C P}\right)$ & $x$ & $\sum$ & $\begin{array}{l}2 \\
1 \\
8 \\
1\end{array}$ & $\begin{array}{l}\text { complex mult. } \\
\text { complex add. } \\
\text { real mult. } \\
\text { real add. }\end{array}$ & $\begin{array}{l}= \\
= \\
= \\
=\end{array}$ & $\left.\begin{array}{r}6 \\
1 / 2 \\
4 \\
1 / 4\end{array}\right)$ \\
\hline
\end{tabular}

6) Compute $\tilde{\mathbf{r}}^{C_{I C I}}(\ell)=\sum_{j=1}^{N_{r}} \tilde{\mathbf{r}}_{j}(\ell)-\tilde{\mathbf{r}}_{j}^{I S I}(\ell)+\mathbf{C}_{j}(\ell)$
Demodulation

symbol will give rise to the data to be introduced in the next iteration of the algorithm.

- Task 7: The demodulation of the signal requires the calculation of the distances to the $Q$ constellation points, where $Q=16$ for 16-QAM and $Q=4$ for 4-QAM. Finding the square distance between any two points requires three real additions and two real multiplications, jointly with one additional $Q$-length minimum index search, giving a total of $3 / 4+2 / 2+17+Q / 2$ cycles for this task.

Overall, the total quantity of cycles necessary to execute one iteration of this interference cancellation algorithm considering $N=64$ subcarriers, 16-QAM, $L=6, L_{\mathrm{CP}}=2$, and two transmit and receive antennas $\left(N_{t}=2\right.$ and $\left.N_{r}=2\right)$ and taking into account the consumption value of $415.8 \mathrm{pW} \cdot \mathrm{s} /$ cycle of the referenced DSP implies an energy consumption, or a saving, of approximately $2.6 \mathrm{~mW}$.

As pointed out above, despite the fact that our proposal implicitly requires the execution of this procedure in the preamble during a fixed number of iterations, the complete procedure, including the data part, will be cost effective in terms of energy provided that the number of data symbols is much higher than the iterations carried out in the preamble.

\section{Simulation Results}

To validate the proposed IJEP in a scenario of insufficient $\mathrm{CP}$, some simulations have been performed. A multi-antenna $2 \times 2$ MIMO-OFDM system consisting of two transmit and two receive antennas has been considered, where the number of subcarriers is $N=64$, and the length of the $\mathrm{CP} L_{\mathrm{CP}}$ is set to one third of the channel length $L$, i.e., $L_{\mathrm{CP}}=L / 3$. The simulated MIMO channels between the antennas are based on individual tapped-delay line models following an exponential profile, which are widely used for implementing the multiple-path channels [20]. It is implicitly assumed that the channel does not vary within the duration of a frame, the different paths between transmit and receive antennas are uncorrelated, and their duration is six taps, i.e., $L=6$. A variable number of frames, with a minimum of 10000 , have been simulated using a 16-QAM modulation scheme, where every frame consists of one preamble and 20 subsequent OFDM symbols corresponding to the data part. Data detection is based on the ML detector on a symbol-by-symbol basis. It is worth noting that these results are obtained without considering any channel-coding scheme. Both the number of subcarriers and the size of the constellation of the modulation scheme have been modified to show the effect of the IJEP in the BER for different systems. For the computation of the FO estimation, the number of rounds of Newton's method was limited to five, and the value of FO is set to $\varepsilon=\{0.2,0.4\}$ to check the independence of the proposal with regard to the FO.

The aim of these simulations is to demonstrate two main aspects of the algorithm. On the one hand, it is shown how the ISI and ICI of the preamble can be iteratively suppressed, outperforming previous proposals and converging to the SCP case regarding the MSE and BER performances. This is achieved using the IJEP presented in Section III. On the other hand, it is proven that this procedure improves the overall performance of the system since the new quasi-free of interference preamble not only reduces the number of subsequent iterations to cancel interferences in the data part but enhances the BER versus the signal-to-noise ratio (SNR) as well.

Summarizing, we will assess the IJEP comparing three different cases, which encompasses the different strategies dealt with so far, by their respective behaviors of MSE and BER.

Case a) Our current proposal, where the IJEP is performed in the $N$ samples of the preamble during a specific number of iterations, denoted by $t_{P}$. The estimations obtained with IJEP execution are subsequently used to cancel the interferences in the data part during other different numbers of iterations, which are denoted by $t_{D}$, to calculate the BER of 


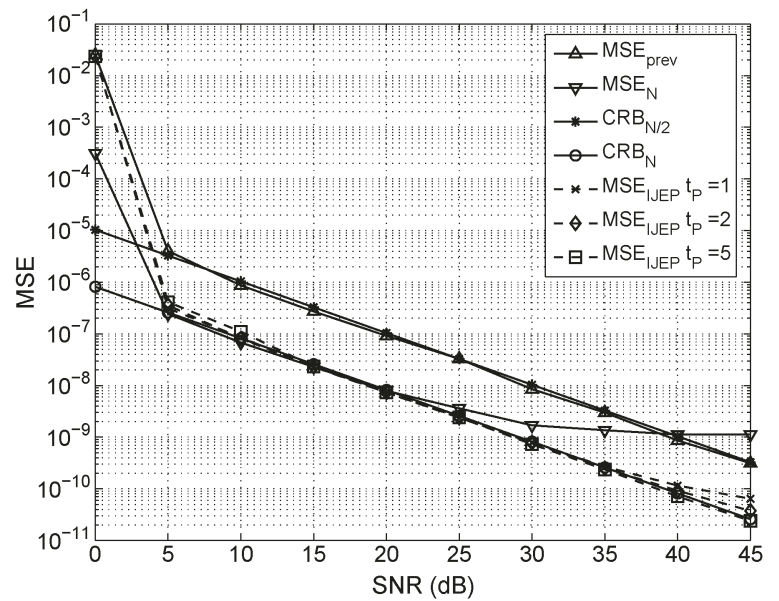

3.1: MSE of FO estimation for $\varepsilon=0.2$

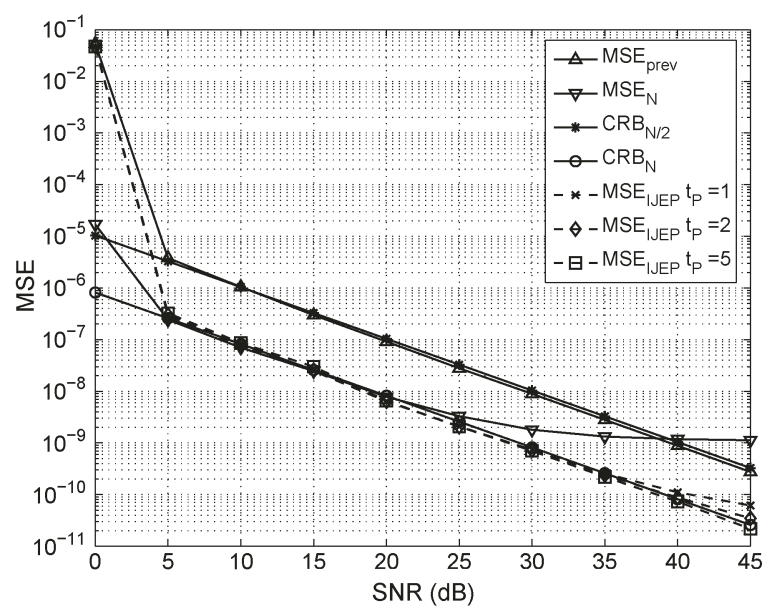

3.3: MSE of FO estimation for $\varepsilon=0.4$

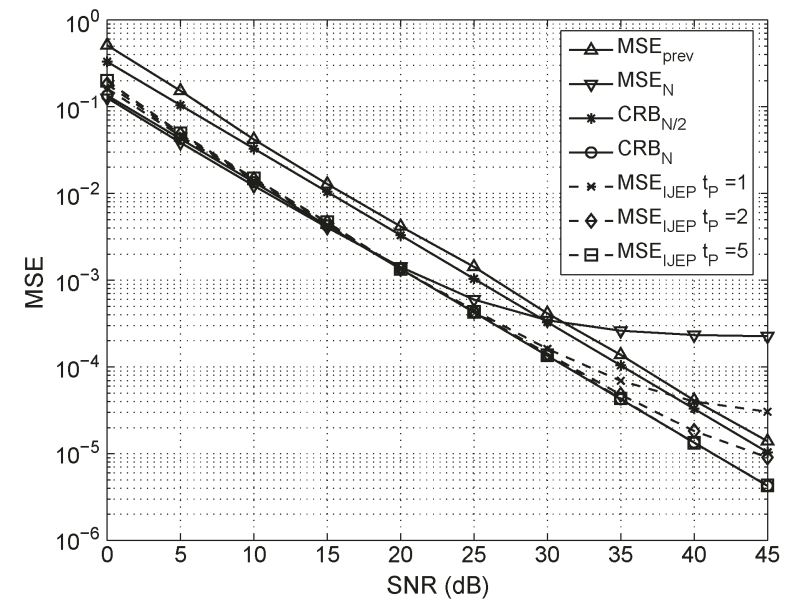

3.2: MSE of channel estimation for $\varepsilon=0.2$

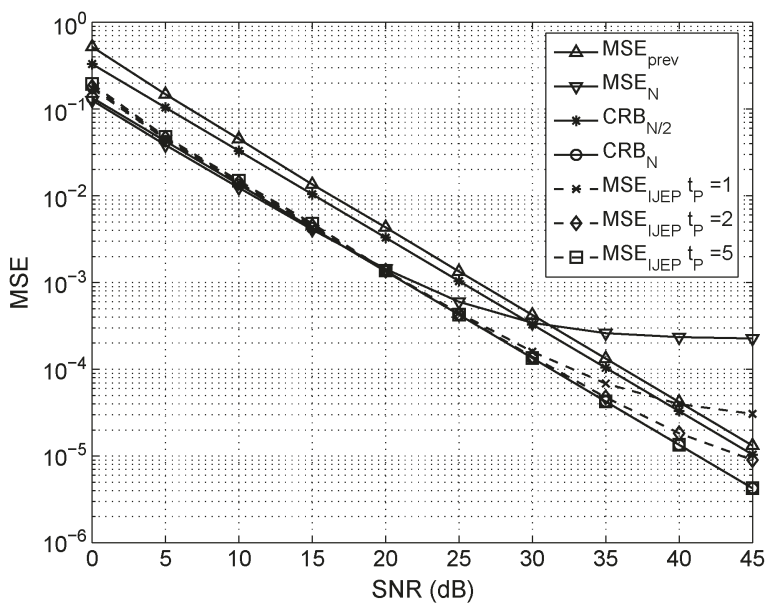

3.4: MSE of channel estimation for $\varepsilon=0.4$

Fig. 3. MSE of FO and channel estimation showing the theoretical CRBs and the different estimation schemes: the current proposal IJEP (Case a, labeled

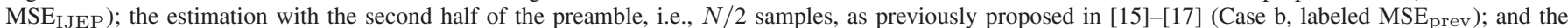
estimation with the whole preamble, i.e., $N$ samples, containing the interferences (Case c, labeled $\mathrm{MSE}_{N}$ ). $16-\mathrm{QAM}, N=64$ subcarriers, $L_{\mathrm{CP}}=2, L=6$, and the number of iterations of IJEP in the preamble $t_{P}=\{1,2,5\}$. (3.1) MSE of FO estimation for $\varepsilon=0.2$. (3.2) MSE of channel estimation for $\varepsilon=0.2$. (3.3) MSE of FO estimation for $\varepsilon=0.4$. (3.4) MSE of channel estimation for $\varepsilon=0.4$.

the system. This case is labeled with the subscript "IJEP" in the figures.

Case b) Procedures disclosed in [15]-[17], where only the second half of the preamble, i.e., $N / 2$ samples that are quasifree of interference, are used to determine the estimations. These less accurate estimations are used, as in the previous case, to later cancel the interferences of the data part and calculate the BER. In this occasion, only the parameter $t_{D}$ applies. This case is labeled with the subscript "prev" in the figures, referring to previous works.

Case c) The estimations are performed with the whole preamble, i.e., $N$ samples, which includes the interferences, but without applying the IJEP. The lack of accuracy of the estimation in this case does not permit the execution of any kind of interference cancellation in the data part. This case is labeled with the subscript " $N$ " in the figures.

The initial assessment is carried out by the performance of the joint channel and FO estimation in comparison with the theoretical bounds given by the CRBs, as exposed in Section IV. The bound will be calculated accounting for the whole preamble ( $N$ samples) as well as considering only the second half $\left(N / 2\right.$ samples). In the figures, $\mathrm{CRB}_{N}$ denotes the bounds for the whole preamble, whereas $\mathrm{CRB}_{N / 2}$ considers only the second half. It must be noted that $\mathrm{CRB}_{N}$ is the bound corresponding to the SCP case.

Figs. 3.1 and 3.2 shows the simulated MSE results for the FO and channel estimations, respectively, for $\varepsilon=0.2$ and, analogously, Figs. 3.3 and 3.4 for the case of $\varepsilon=0.4$. For both cases, the performance of the estimators using the whole preamble but without the IJEP, which encompasses the interferences (Case c, $\mathrm{MSE}_{N}$ curves), drives to an error floor in the medium-to-high SNR region, but it converges to $\mathrm{CRB}_{N}$ for low SNR values. These curves in both cases are clearly improved in the mediumto-high SNR interval when only the second half of the preamble is used for the estimation (Case b, MSE $E_{\text {prev }}$ curves), since the estimations converge to the theoretical CRB calculated for the second half of the preamble $\left(\mathrm{CRB}_{N / 2}\right)$.

It is worth noting a particular aspect shown in Figs. 3.2 and 3.4. The curve corresponding to the simulated MSE of the channel estimation obtained with the second half of the preamble (Case b, $\mathrm{MSE}_{\text {prev }}$ curve) does not exactly converge to $\mathrm{CRB}_{N / 2}$. This is due to the error introduced by an FO estimation obtained with the second half of the preamble instead of with the whole preamble. 


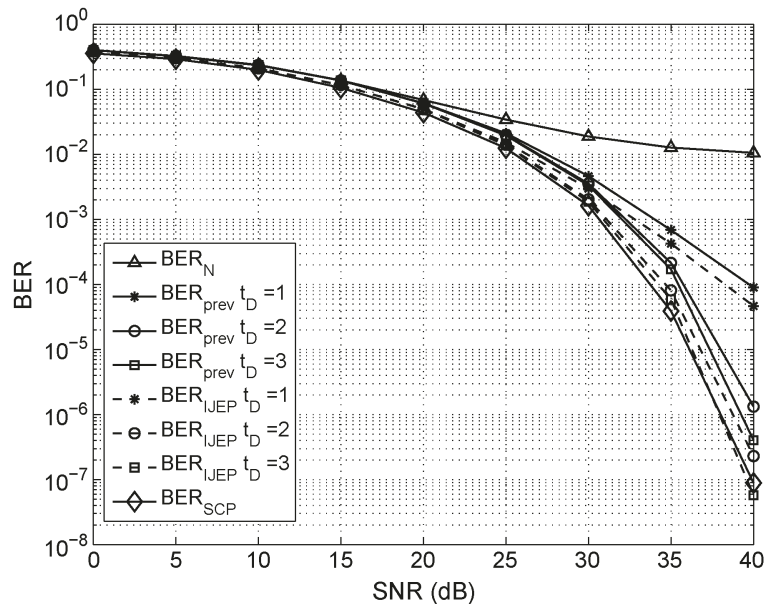

4.1: 16-QAM, $N=64$ sub-carriers

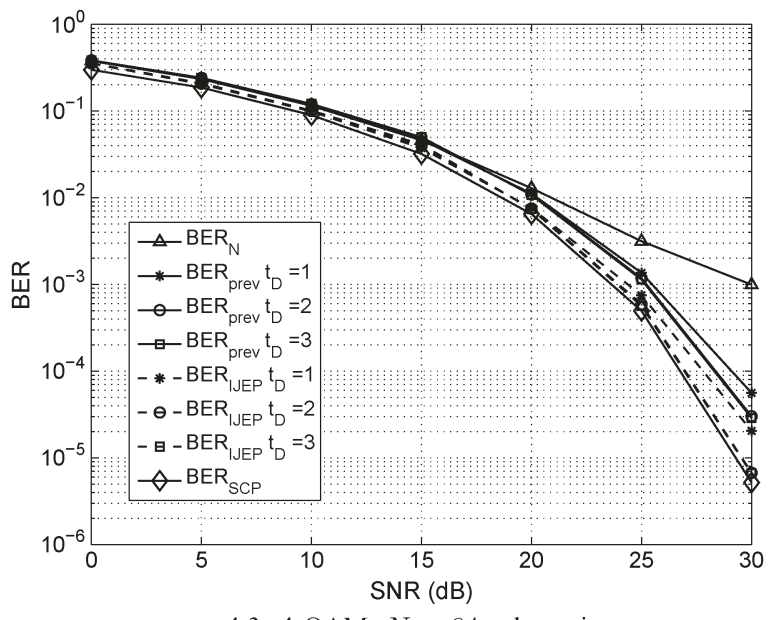

4.3: 4-QAM, $N=64$ sub-carriers

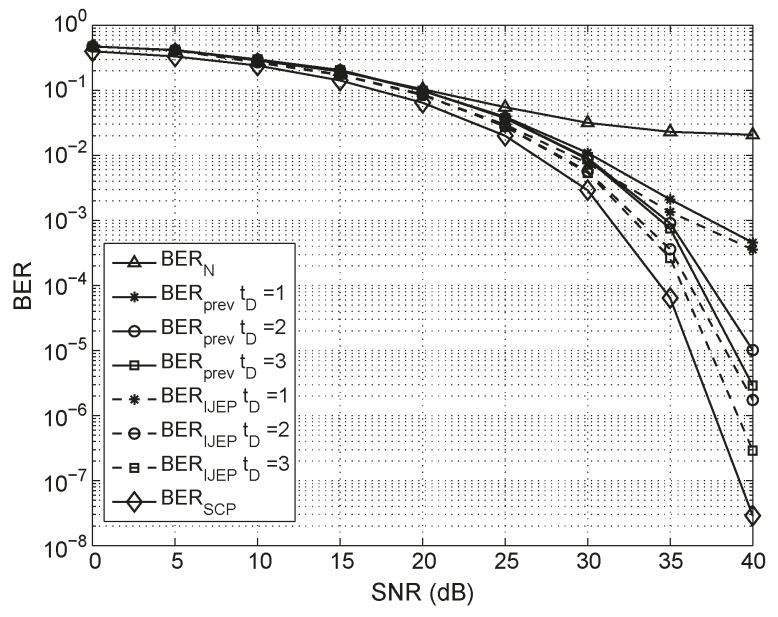

4.2: 16-QAM, $N=32$ sub-carriers

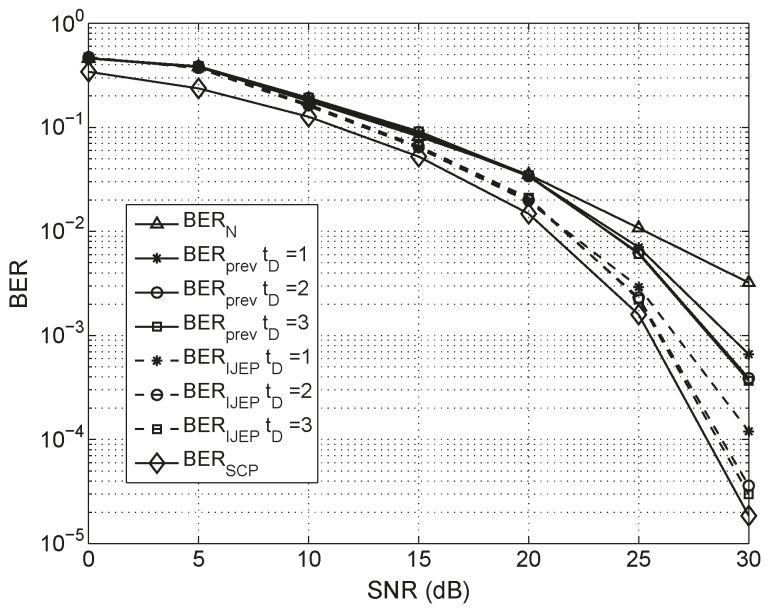

4.4: 4-QAM, $N=32$ sub-carriers

Fig. 4. BER of the system with interference cancellation in the data part utilizing the different estimation schemes: the current proposal IJEP (Case a, labeled

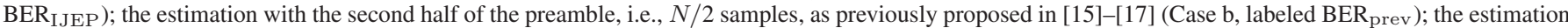
with the whole preamble, i.e., $N$ samples, without canceling the interferences $\left(\right.$ Case c, labeled $\left.\mathrm{BER}_{N}\right)$; and the sufficient $\mathrm{CP}$ case $\left(\right.$ labeled $\mathrm{BER}$ SCP). $L_{\mathrm{CP}}=2$, $L=6, \varepsilon=0.4$, and the number of iterations of the interference cancellation in the data part $t_{D}=\{1,2,3\}$. (4.1) 16-QAM, $N=64$ subcarriers. (4.2) 16-QAM, $N=32$ subcarriers. (4.3) 4-QAM, $N=64$ subcarriers. (4.4) 4-QAM, $N=32$ subcarriers.

These four figures also depict how the curves are progressively approaching to the CRB of the whole preamble $\left(\mathrm{CRB}_{N}\right)$ as the number of iterations $t_{P}$ of the IJEP increases (Case a, $\mathrm{MSE}_{\mathrm{IJEP}}$ curves). This indicates that the process of interference suppression consecutively eliminates the distortion allocated in the first part of the preamble and that it is independent of the FO value, provided that the FO is within the acquisition range corresponding to the type of sequence used in the preamble. After the target value of $t_{P}=5$ is reached, we attain the final joint FO and channel estimation. The performance of these last estimations is closer to the corresponding bounds as a function of the computed iterations, since the higher the number of iterations, the closer the MSE IJEP curves to the $\mathrm{CRB}_{N}$.

On the other hand, the comparison of the BER as a function of the SNR is initially shown in Fig. 4.1 for $N=64$ subcarriers and 16-QAM. It is considered that the OFDM symbols in the data part of the frame also suffer the effects of an insufficient $\mathrm{CP}$, as in the preamble, and a number of up to $t_{D}=3$ iterations for the interference cancellation have been selected. It is shown how the BER corresponding to Case c $\left(\mathrm{BER}_{N}\right.$ curve) leads to an error floor in the medium-to-high SNR region. Additionally, it is easy to observe how the curves corresponding to our proposal in Case a $\left(\mathrm{BER}_{\mathrm{IJEP}}\right.$ curves) clearly overcome the previous procedure specified in Case $\mathrm{b}$ ( $\mathrm{BER}_{\text {prev }}$ curves) and converge to the ideal situation represented by the SCP scenario $\left(\right.$ BER $_{\mathrm{SCP}}$ curves).

It is clearly seen that for a target value of the BER, this is reached with less number of iterations if the IJEP is performed, e.g., in Fig. 4.1, the point belonging to Case $\mathrm{b}$ and $t_{D}=3$ iterations for a BER value of $4 \cdot 10^{-7}$ and $\mathrm{SNR}=40 \mathrm{~dB}$ is improved with $t_{D}=2$ iterations for Case a. Hence, we can conclude that, in the medium-to-high SNR region, the IJEP is more effective and lead to a computational cost saving of one iteration for each data symbol within the frame. This is particularly important since this simple saving means less power consumption and, consequently, a considerable improvement in the battery duration. In addition, it is shown that after several iterations, the ideal limit, which is represented by the BER $_{\mathrm{SCP}}$ curve, is reached.

To complete the assessment of the IJEP, three additional simulations have been conducted by varying the initial parameters of the system. For instance, in Fig. 4.2, the BER belonging to 
16-QAM and $N=32$ subcarriers is depicted. Following the same directives than in the previous figure, a similar behavior that gives an enhancement of one iteration to our proposal with regard to previous procedures is observed. Similarly, Figs. 4.3 and 4.4 shows the BER for 4-QAM and $N=64$ and $N=32$ subcarriers, respectively. Fig. 4.3 shows analogous results, but Fig. 4.4 shows even better results with an improvement of three iterations. Obviously, this leads to an outstanding saving of the battery.

\section{CONCLUSION}

A new proposal named IJEP is addressed to improve the joint channel and FO estimation in MIMO-OFDM systems in the presence of ISI-ICI in the preamble due to highly disruptive channels, where the $\mathrm{CP}$ can be shorter than the channel length or because of the partial or total suppression of the CP to augment the system capacity. It has been shown that using an SS as preamble, its non-distorted part (second half) permits obtaining an initial FO and channel estimation that is able to cancel the existing interference in the distorted part (first half). It has been proven that the interference cancellation process in the preamble drives to new estimators computed with the whole preamble and whose performances are similar to the ideal case of a sufficient $\mathrm{CP}$, converging to the theoretical CRB and only depending on the number of iterations performed. This implies that the number of iterations necessary to cancel the interference in the data part, which is also affected by an insufficient $\mathrm{CP}$, decreases as a function of the iterations computed in the preamble. This dramatically reduces the computational cost and consequently extends the battery life duration. Moreover, it has been proven how the BER performance is enhanced when the IJEP is used in the preamble, which approaches to the bound given by the SCP scenario.

\section{REFERENCES}

[1] Digital Video Broadcasting (DVB); Transmission System for Handheld Terminals (DVB-H), ETSI EN 302304 V1.1.1, Nov. 2004.

[2] Parameter values for the HDTV standards for production and international programme exchange, ITU-R BT.709-5, Apr. 2004.

[3] N. Itoh and K. Tsuchida, "HDTV mobile reception in automobiles," Proc. IEEE, vol. 94, no. 1, pp. 274-280, Jan. 2006.

[4] A. F. Molisch, Wireless Communications, 2nd ed. Hoboken, NJ, USA: Wiley, 2011.

[5] D. Rauschmayer, ADSL/Vdsl Principles: A Practical and Precise Study of Asymmetric Digital Subscriber Lines and Very High Speed Digital Subscriber Lines. Chambly, QC, Canada: Alpel, 1998.

[6] F. Bader and N. Zorba, Advances on Processing for Multiple Carrier Schemes: OFDM and OFDMA. Commack, NY, USA: Nova, 2012, ser. Computer Science, Technology and Applications.

[7] A. Goldsmith, S. A. Jafar, N. Jindal, and S. Vishwanath, "Capacity limits of MIMO channels," IEEE J. Sel. Areas Commun., vol. 21, no. 5, pp. 684702, Jun. 2003.

[8] S. Chen and C. Zhu, "ICI and ISI analysis and mitigation for OFDM systems with insufficient cyclic prefix in time-varying channels," IEEE Trans. Consum. Electron., vol. 50, no. 1, pp. 78-83, Feb. 2004.

[9] J. Lim, C. Choi, and G. Im, "MIMO-OFDM with insufficient cyclic prefix," IEEE Commun. Lett., vol. 10, no. 5, pp. 356-358, May 2006.

[10] A. F. Molisch, M. Toeltsch, and S. Vermani, "Iterative methods for cancellation of intercarrier in OFDM systems," IEEE Trans. Veh. Technol., vol. 56, no. 4, pp. 2158-2167, Jul. 2007.

[11] M. Toeltsch and A. F. Molisch, "Efficient OFDM transmission without cyclic prefix over frequency-selective channels," in Proc. 11th IEEE Int. Symp. PIMRC, 2000, vol. 2, pp. 1363-1367.
[12] U. Yunus, L. H. Lin, and K. Yamashita, "A novel equalization method for OFDM systems without guard interval," in Proc. IEEE GLOBECOM, 2010, pp. 1-5.

[13] L. Yang and S. Cheng, "Optimum ISI and ICI cancellation filter for OFDM systems without CP," IEICE Trans. Commun., vol. E90-B, no. 2, pp. 364-367, Jan. 2007.

[14] Digital Video Broadcasting (DVB); Framing Structure, Channel Coding and Modulation for Digital Terrestrial Television, ETSI EN 300744 v1.5.1, Nov. 2004.

[15] D. Kim and G. L. Stüber, "Residual ISI cancellation for OFDM with applications to HDTV broadcasting," IEEE J. Sel. Areas Commun., vol. 16, no. 8, pp. $1590-1599$, Oct. 1998.

[16] V. Nguyen, M. Pätzold, F. Maehara, H. Haas, and M. Pham, "Channel estimation and interference cancellation for MIMO-OFDM systems," IEICE Trans. Commun., vol. E-90-B, no. 2, pp. 277-290, Feb. 2007.

[17] C. Prieto del Amo, V. P. Gil-Jiménez, and M. J. Fernández-Getino García, "Joint channel and frequency offset estimation in MIMO-OFDM systems with insufficient cyclic prefix," Phys. Commun., vol. 4, no. 4, pp. 254 265, Dec. 2011.

[18] IEEE Standard for Local and Metropolitan area networks Part 16: Air Interface for Fixed Broadband Wireless Access Systems, IEEE Std. 802.162009, 2009

[19] D. Serre, Matrices: Theory and Applications. New York, NY, USA: Springer-Verlag, 2002

[20] Y. S. Choo, J. Kim, W. Y. Yang, and C. G. Kang, MIMO-OFDM Wireless Communications with Matlab. Singapore: Pte Ltd., 2010.

[21] R. J. Baxley and N. Chen, "Power savings analysis of peak-to-average power ratio reduction in OFDM," IEEE Trans. Consum. Electron., vol. 50, no. 3, pp. 792-798, Aug. 2004

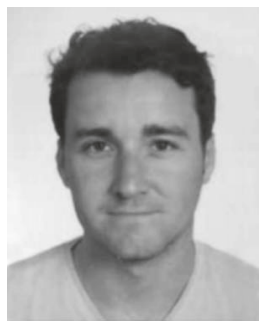

Carlos Prieto del Amo received the B.S. degree in telecommunication engineering from the Polytechnic University of Madrid, Madrid, Spain, in 1996 and the M.Eng. degree in telecommunication engineering from Carlos III University of Madrid, Leganés, Spain, in 2001. He is currently working toward the $\mathrm{Ph} . \mathrm{D}$. degree with the Department of Signal Theory and Communications, Carlos III University of Madrid.

For five years, he was with Rohde \& Schwarz Spain as a Project Manager on radio communication systems. Next, he was with SIDSA for one year, working on the research of xDSL technologies. He is currently at Airbus Military, Madrid, as an Electromagnetic Compatibility Engineer.

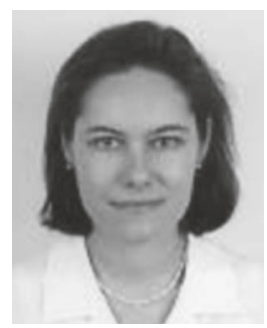

M. Julia Fernández-Getino García (S'99-AM'02M'03) received the M. Eng. and Ph.D. degrees in telecommunication engineering from the Polytechnic University of Madrid, Madrid, Spain, in 1996 and 2001, respectively.

She is currently with the Department of Signal Theory and Communications, Carlos III University of Madrid, Leganés, Spain, as an Associate Professor. From 1996 to 2001, she held a research position with the Department of Signals, Systems and Radiocommunications, Polytechnic University of Madrid. She visited Bell Laboratories, Murray Hill, NJ, in 1998; visited Lund University, Lund, Sweden, during two periods in 1999 and 2000; visited Politecnico di Torino, Torino, Italy, in 2003 and 2004; and visited Aveiro University, Aveiro, Portugal, in 2009 and 2010. Her research interests include multicarrier communications, coding, and signal processing for wireless systems.

Prof. Fernández-Getino García received the best "Master Thesis" and "Ph.D. Thesis" awards from the Professional Association of Telecommunication Engineers of Spain in 1998 and 2003, respectively; the "Student Paper Award" and the "Certificate of Appreciation" at the 1999 IEEE International Symposium on Personal, Indoor, and Mobile Radio Communications and the 2000 IEEE Vehicular Technology Conference, respectively; the "Ph.D. Extraordinary Award" from the Polytechnic University of Madrid in 2004; and the "Excellence Award" from Carlos III University of Madrid in 2012 for her research. 